Ephemeride for $x 2^{\text {h }}$ M. Z. Berlin.

\begin{tabular}{|c|c|c|c|c|c|c|c|c|c|c|c|c|c|c|}
\hline \multirow{2}{*}{$\frac{1894}{\text { Febr. } 16}$} & \multicolumn{2}{|r|}{$\alpha$ app. } & \multicolumn{2}{|c|}{$d$ app. } & $\log \Delta$ & Ab.-Z. & \multicolumn{2}{|c|}{1894} & \multicolumn{2}{|r|}{$\alpha$ app. } & \multicolumn{2}{|c|}{ d app. } & $\log \Delta$ & Ab. $-Z$ \\
\hline & & ${ }^{b} 22^{m} 47^{3} 5^{8}$ & $+14^{\circ} 21$ & $x^{\prime} 14: 3$ & 0.339723 & $18^{\mathrm{m}} 10^{s}$ & März & 8 & $11^{\mathrm{h}}$ & $6^{\mathrm{m}} 24^{\mathrm{s}} \cdot 44$ & $+15^{\circ} 4$ & $42^{\prime} 17^{\prime \prime} 4$ & 0.335274 & $17^{\mathrm{m}} 59^{\mathrm{s}}$ \\
\hline 17 & & $22 \quad 3 \cdot 32$ & .1425 & 541.5 & 338955 & 8 & & 9 & & 533.36 & 154 & $45 \quad 32.2$ & 335674 & 180 \\
\hline 18 & & $21,8.27$ & 1430 & 308.0 & $33824 I$ & 7 & & 10 & & $4 \quad 42.49$ & 154 & $\begin{array}{ll}8 & 40.3\end{array}$ & $33^{6131}$ & $I$ \\
\hline 19 & & $20 \quad 32.48$ & 143 & $\begin{array}{ll}34 & 33.5\end{array}$ & $3375^{82}$ & 5 & & 11 & & $\begin{array}{l}351.88 \\
\end{array}$ & 155 & 5141.5 & $33^{6648}$ & 3 \\
\hline 20 & & 1945.99 & 1438 & $\begin{array}{ll}8 & 57.7\end{array}$ & 336978 & 3 & & 12 & & $3 \quad 1.58$ & 155 & 5435.6 & 337220 & 4 \\
\hline 21 & & 1858.83 & 144 & 320.5 & 336431 & 2 & & 13 & & 211.65 & 155 & $57 \quad 22.3$ & 51 & 6 \\
\hline 22 & & 1811.05 & 144 & 1741.4 & $33594 \mathrm{I}$ & I & & 14 & & 122.14 & 16 & $0 \quad 1.5$ & 38536 & 7 \\
\hline 23 & & 1722.70 & 145 & $; 20.1$ & 335 & 180 & & 15 & in & - 33.09 & 16 & 233.0 & 339278 & 9 \\
\hline 24 & & 1633.83 & 145 & $; 6 \quad 16.2$ & 335 & 1759 & & 16 & 10 & $59 \quad 44.54$ & 16 & $4 \quad 56.7$ & 340073 & I I \\
\hline 25 & & $15 \quad 44.47$ & 15 & $\circ \quad 29.6$ & 334817 & 58 & & 17 & & $5^{8} \quad 5^{6.56}$ & 16 & $\begin{array}{ll}7 & 12.4\end{array}$ & 340922 & 13 \\
\hline 26 & & 1454.68 & 15. & $439 . i$ & 334560 & 57 & & 18 & & $\begin{array}{ll}58 & 9.18\end{array}$ & 16 & 920.0 & 823 & 16 \\
\hline 27 & & $14 \quad 4.51$ & 158 & $8 \quad 46: 4$ & 2 & 5 & & 19 & & 5722.43 & 161 & 11195 & 342778 & 18 \\
\hline 28 & & 1314.01 & 1512 & 1249.2 & 334224 & 57 & & 20 & & $\begin{array}{ll}56 & 36.37\end{array}$ & $16 \mathrm{I}$ & 310.6 & 343782 & 20 \\
\hline Mărz I I & & $12 \quad 23.23$ & 1518 & $\begin{array}{ll}6 \quad 47.9 \\
6\end{array}$ & 334146 & $5^{6}$ & & $2 \mathrm{I}$ & & $55 \quad 51.03$ & 161 & 1453.3 & 344837 & 23 \\
\hline 2 & & $1 \mathrm{I} \cdot 32.22$ & 1520 & 2042.1 & 7 & $5^{6}$ & & 22 & & $55 \quad 6.46$ & 161 & 1627.6 & 940 & 26 \\
\hline 3 & & 1041.04 & 152 & $24 \quad 31.6$ & 334169 & 56 & & 23 & & $54 \quad 22.68$ & 161 & 753.3 & 347092 & 29 \\
\hline 4 & & 949.75 & 1528 & $28 \quad 16.0$ & 334270 & 57 & & 24 & & $53 \quad 39.73$ & 161 & 1910.5 & 348290 & 32 \\
\hline 5 & & $\begin{array}{ll}8 & 58.39\end{array}$ & 153 & 3155.1 & & 5 & & 25 & & 5257.66 & 162 & $20 \quad 19.0$ & 349535 & 35 \\
\hline 86 & & $8 \quad 7.01$ & 153 & $\begin{array}{ll}35 & 28.6\end{array}$ & 334653 & 58 & & 26 & & $52 \quad 16.50$ & 162 & $\begin{array}{ll}21 & 18.8\end{array}$ & 350825 & 38 \\
\hline 7 & & $7 \quad 15.68$ & 153 & $3^{8} \quad 5^{6 . x}$ & 334934 & 58 & & 27 & & 5136.26 & 162 & $22 \quad 9.9$ & 352159 & 42 \\
\hline 8 & 11 & 624.44 & +154 & $42 \quad 17.4$ & 0.335274 & 1759 & & 28 & 10 & 50 56.98 & +162 & 2252.2 & 0.353536 & 1885 \\
\hline
\end{tabular}

Opposition in AR. März 6 ; Grösse 9 m. Reduction auf $1855.0: \Delta \alpha=-2^{\mathrm{m}} 3^{\mathrm{s}}, \Delta \delta=+12: 7$.

Karlsruhe 1894 Febr. 3.

F. Ristenpart.

The Perseids on the $10^{\text {th }}$ August 1893.

Shooting stars were watched at the Hongkong Obser- $\mid$ (omitting one very long path) was $19^{\circ}$. The average vatory between $9^{b} 5^{\mathrm{m}} \mathrm{p}$. m. M. T. Hongkong on the $10^{\text {th }}$, duration was $1 / 2$ sec. One was visible during 3 sec. and $2^{\mathrm{h}} 25^{\mathrm{m}}$ a. $\mathrm{m}$. on the $\mathrm{II}^{\mathrm{th}}$. The radiant was determined (length of path about $15^{\circ}$ at $8^{\mathrm{h}} 8^{\mathrm{m}} \mathrm{p}$. $\mathrm{m}$.), I during $\mathrm{I}$ sec., as $42^{\circ}, 55^{\circ}$ at II p. m. and $44^{1} \frac{1}{2}^{\circ}, 5812^{\circ}$ at 2 a. m. ; or I during $3 / 4$ sec., 6 during $1 / 2$ sec., 7 during $1 / 3$ sec., as $43^{\circ} 40^{\prime}, 57^{\circ} 20^{\circ}$ (referred to the equinox for 1855 ) at 5 during $1 / 4$ sec., and 2 during $1 / 5$ sec. Ten showed white 1 a. $\mathrm{m}$. on the $\mathrm{I}^{\text {th }}$ from all the observations. There were streaks. All the shooting stars were white except 3 which 3 larger than the $1^{\text {st }}$ magnitude, 2 of the ${ }^{\text {tot }}, 7$ of the appeared very near the radiant, and were deep yellow. Five $2^{\text {nd }}, 6$ of the $3^{\text {sd }}, 3$ of the $4^{\text {th }}$, and 2 of the $5^{\text {th }}$. The shooting stars were noted from different radiants. They were average was 2.4 magnitude. The average length of path all white and some of them left streaks.

Hongkong Observatory 1893 November.

W. Doberck.

\title{
Beobachtung des neuen Sterns in Norma.
}

Die Centralstelle erhielt am 15. Febr. $3^{\mathrm{h}}$ Morgens folgendes Telegramm aus Cambridge Mass.:

- Holden telegraphs: Nova Normae was observed by Campbell, Lick Observatory, February I 3 , magnitude 9.5; spectrum four bright lines, same relative intensity and position as Nova Aurigae August 1892, hence nebulous.

Das Telegramm wurde an die Mitglieder der Centralstelle, sowie an die Sternwarten der Südhalbkugel weiter befördert.

Kiel 1894 Febr. 15.

$K r$.

\section{Inhalt:}

Zu Nr. 3211 . R. Luther. Kreismikrometer-Beobachtungen von kleinen Planeten am Siebenfuisser zu Düsseldorf. 297. - E. Harkzeig. Ortsbestimmungen und Mittheilungen zu neueren veränderlichen Sternen. 305. - W. Huggiss. On the visual appearance of Nova (T) Aurigae. 309. - F. Riskenpart. Elemente und Ephemeride des Planeten (349) Dembowska. 309. - W. Doberck. The Perseids

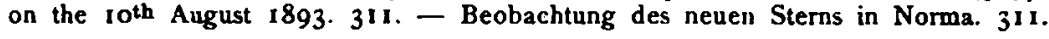

